\title{
Unified Satellite and Terrestrial ACM Design
}

\author{
Alireza Haqiqatnejad and Farbod Kayhan \\ Interdisciplinary Centre for Security, Reliability and Trust (SnT), \\ University of Luxembourg \\ (emails: \{alireza.haqiqatnejad, farbod.kayhan\}@uni.lu).
}

\begin{abstract}
First step towards integrating satellite and terrestrial standards at the physical layer is to design a unified packet frame structure, and in particular, a unified adaptive coding and modulation (ACM) scheme without substantial loss in either of the systems. In this paper we introduce a methodology to design such a set of modulation and coding (MODCOD) combinations. In the first step, we design a set of base MODCODs for the AWGN channel. Then, for any other given channel model, we build a new set of MODCODs through a suitable transformation of the base MODCODs. We mainly focus on two types of channels: (a) non-linear satellite channels, and (b) AWGN channels with fading. We compare our results with latest digital video broadcasting standards, namely, DVB-T2 and DVB-S2X.
\end{abstract}

\section{Nomenclature}

$\begin{array}{ll}\text { ACM } & \text { adaptive coding and modulation } \\ \text { APSK } & \text { amplitude and phase-shift keying } \\ \text { AWGN } & \text { additive white Gaussian noise } \\ \text { BER } & \text { bit error rate } \\ \text { DVB } & \text { digital video broadcasting } \\ \text { HPA } & \text { high power amplifier } \\ \text { LDPC } & \text { low-density parity-check } \\ \text { MODCOD } & \text { modulation and coding } \\ \text { PAM } & \text { pulse amplitude modulation } \\ \text { PAMI } & \text { pragmatic average mutual information } \\ \text { QAM } & \text { quadrature amplitude modulation } \\ \text { QCI } & \text { QAM to circular isomorphic } \\ \text { (P)SNR } & \text { (peak) signal to noise ratio }\end{array}$

\section{Introduction}

Real-time adaptation of transmission parameters according to channel conditions is one of the main features of the ever growing high-throughput communication systems. Time and/or user varying channel condition is an inherent characteristic of many communication systems such as satellite, cellular networks and broadcast systems. ACM schemes are frequently used in such systems to provide significant capacity gains by allowing the transmission format to be changed, depending on the application and/or channel quality. By employing an ACM scheme, the transmitter is able to switch between several MODCODs, choosing the largest available modulation and code rate which ensures a target detection error rate, and thus providing the maximum reliable spectral efficiency to each user. ACMs have been adopted in several standards such as DVB and CCSDS. ${ }^{1-3}$ However, the chosen coding and modulation schemes are usually different from one standard to another.

Typically, in ACM schemes, all MODCODs are predefined and known to both transmitter and receiver. Several factors affect designing of the MODCODs for a given system, including noise model (AWGN, phase noise,... ), channel model (fading, linear, non linear, ...), operating band (Ka band, X band,...), target error rate, and target spectral efficiency. While the coding scheme is not usually affected by these parame- 
ters, poor choice of the modulation set may result in a significant performance loss. In particular, it is known that circular APSK constellations perform much better than QAM constellations over satellite channels with HPAs on board. ${ }^{4}$ This is mainly due to the non-linear characteristics of HPAs near the saturation point.

Although ACM schemes provide remarkable benefits to a communication system in terms of capacity and transmission flexibility, they inevitably increase the complexity of the transmitter and the receiver as well as imposing some overhead on the transmitted frames. In fact, the larger the number of predefined MODCODs, the higher the additional complexity and the amount of overhead will be. Some of the current ACM schemes use over 100 MODCODs, and even higher number of MODCODs may be needed in the next generations due to higher granularity requirements.

In general, a high order constellation set that performs well over a wide range of channel models is not known in the literature. Some families of APSK constellations, namely star-shaped APSK, are shown to achieve the capacity of AWGN channel. ${ }^{5}$ However, the convergence rate as a function of constellation size is very slow and often does not provide any gain with respect to DVB-S2X APSK and even QAM constellations in the SNR range of interest. On the other hand, the constellations optimized over linear channels, such as non-uniform QAM, show a rather large gap from the peak-power limit capacity. ${ }^{6}$ To resolve these problems, for example in DVB-S2X, some of the MODCODs are designed specifically for the use over the linear channel.

In this paper we propose a different approach. The main idea is to introduce a set of base MODCODs optimized over the AWGN channel, and then modify this set for the use over other channel models. We choose the optimized non-uniform QAM constellations as the set of base MODCODs. The MODCODs over non-linear and fading channels are related to the base set through a transformation applied on the constellations. In particular, we use the radial mapping over the non-linear channel, ${ }^{7,8}$ and the constellation rotation over the fading channel. ${ }^{9}$ Targeting high data rate MODCODs, we confine ourselves to 16, 64 and 256 -ary constellations. We present both the average mutual information and BER simulation results for the proposed ACM scheme, and compare them with DVB-S2X and DVB-T2 standards. Our results show that the overall loss with respect to DVB-S2X, where MODCODs are separately designed for both linear and nonlinear channels, is around $0.2 \mathrm{~dB}$ in the worst case.

Beside unifying the MODCODs for different standards, our results indicate that fewer MODCODs may be needed compared to the current standards without sacrificing the performance. For example, in the current DVB-S2X standard, some MODCODs are dedicated only to the linear channel and some others to the non-linear channel. These two sets of MODCODs can be merged by introducing an additional block in the communication chain. In our scheme, these MODCODs are related to each other through a deterministic transformation.

The rest of this paper is organized as follows. We describe the notations and present our system model in Section II. This is followed by defining the channel-adapted transformation block. In Section III, we provide the mutual information analysis for various constellations of interest. We present the simulation results in Section IV and compare the BER of the proposed ACM scheme with those of DVB-S2X and DVB-T2. Finally, we conclude the paper in Section V.

\section{System Model}

A conventional ACM scheme, as shown in Figure 1, is able to switch between a number of MODCODs as a function of the instantaneous channel state and the target spectral efficiency. ${ }^{10}$ The MODCOD is selected based on the channel estimation at the receiver which is available to the transmitter using a finite-rate feedback channel. Depending on the application and the wireless environment, a sufficiently wide range of received SNRs is divided into several intervals, each of which is assigned a MODCOD with respect to the spectral efficiency requirements. Therefore, any given spectral efficiency is supported by changing either the number of bits per modulated symbol or the coding rate. However, in general, the same MODCODs cannot be used for different channel models (e.g. linear/non-linear), and therefore, separate MODCOD sets are needed for each channel model which leads to increasing the total number of MODCODs. ${ }^{11}$

Our goal is to propose an ACM scheme that can be adapted to different channel models. This is done by first defining a set of base MODCODs and then modifying this set as a function of channel model using a deterministic transformation. In other words, the MODCOD set for any given channel model is related to the base MODCOD set through an additional system block, which is referred to as channel-adapted transformation (CAT). As we will see, the CAT block is basically a multicriteria deterministic function. The proposed scheme is illustrated in Figure 2, where the MODCOD set and the CAT functionality are selected 


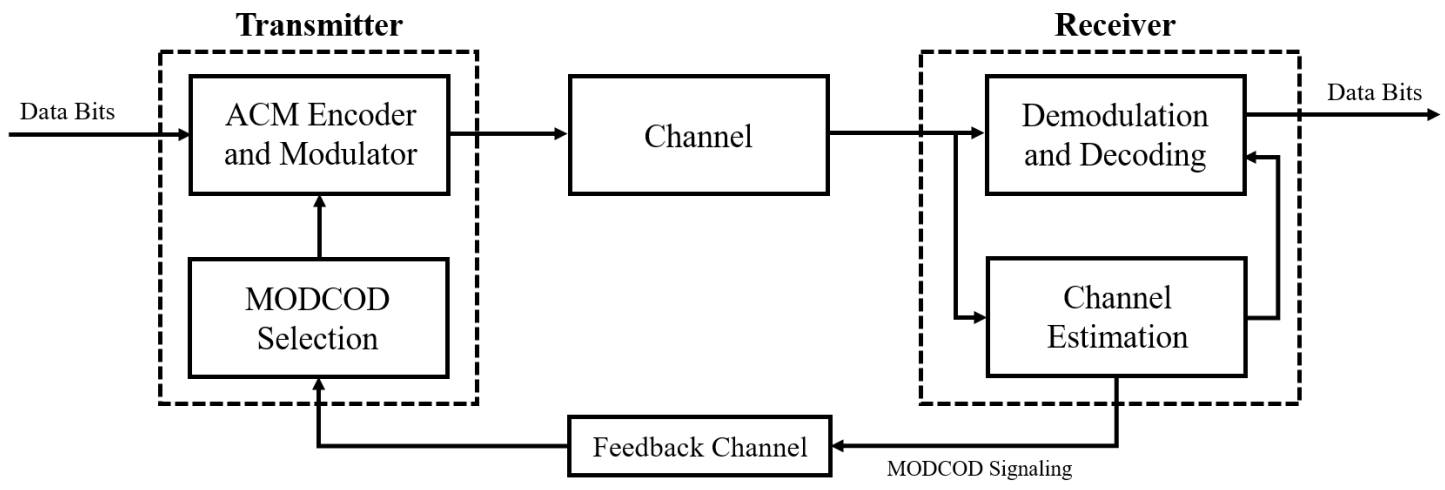

Figure 1. Simplified block diagram of the conventional ACM scheme.

based on the information provided by the receiver through a feedback channel. This model can be readily applied to any ACM communication system by proper designing of base MODCODs and the CAT block.

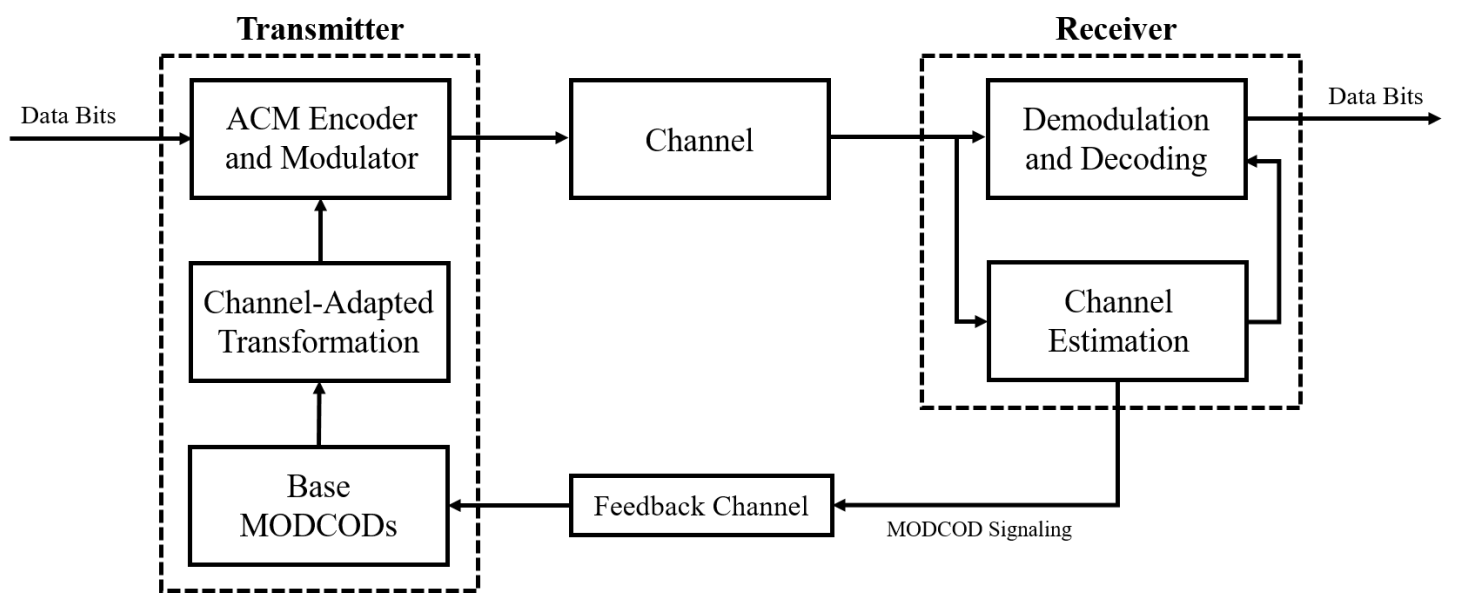

Figure 2. Block diagram of the proposed unified ACM scheme with channel-adapted MODCOD transformation.

In what follows, we study the possibility of finding the base MODCOD set for the linear channel being adaptable to other channel models through the CAT block. It is important to notice that one may define different base MODCODs for another channel model which will result in a different CAT block.

\section{A. Base MODCOD set}

The first step in designing the unified ACM is to find the base MODCOD set. In this paper we only define the modulation scheme to be used in each MODCOD, and therefore we do not change the coding scheme. The MODCODs in DVB-S2X and DVB-T2 standards cannot be interchangeably used without a substantial loss in one or the other system. The DVB-S2X standard mainly adopts APSK constellations in the MODCODs targeted for non-linear channels. In general, APSK constellations are not optimal over linear channels. Therefore, some MODCODs in DVB-S2X are specifically designed for use over linear channels. ${ }^{1}$ On the other hand, DVB-T2 uses QAM constellations in its MODCODs, but it is well known that the QAM signaling leads to noticeable performance loss over non-linear channels. ${ }^{12}$ The opposing performance of these MODCODs over different channel models leads us to design channel-adapted MODCODs with competitive performance over both linear and non-linear channels.

Non-uniform QAM constellations have been studied by several authors due to their potential shaping gain and higher mutual information with respect to the conventional uniform QAM over the AWGN channel. ${ }^{6,13-16}$ These constellations also allow for a low complexity detection, as they can be obtained by the Cartesian product of two non-uniform PAM constellations. This family of QAM constellations is considered to be 
employed in ATSC 3.0 broadcasting standard. ${ }^{17}$ The underlying idea is to use equiprobable symbols with unequal spacing, which results in approximately Gaussian input distribution at high SNR. ${ }^{14}$ It should be noted that finite non-uniform QAM constellations are not the best known constellations over AWGN channel when the input-output mutual information is considered. ${ }^{18,19}$

A method for optimizing non-uniform QAM constellations by maximizing the mutual information over the AWGN channel is presented in. ${ }^{13}$ Taking into account the quadrant symmetry of QAM, this optimization can be simplified to only finding the optimal positive amplitudes of a PAM constellation (i.e., 1D optimization). For a square $M$-QAM constellation, this leads to have a set of $\sqrt{M} / 2$ PAM symbols to be optimized. The Cartesian product of this set with itself gives the constellation points on the first quadrant, and the remaining points can be easily constructed by the quadrant symmetry. In an ACM system, the non-uniform QAM constellations to be used in different MODCODs need to be optimized separately for each SNR range.

In this paper we adopt non-uniform QAM constellations, obtained by optimization over the linear AWGN channel, as the set of base MODCODs. We refer to these constellations as non-uniform (NU) QAM for simplicity. We use the same optimization method as in, ${ }^{20}$ i.e., the simulated annealing algorithm, to perform 1D optimization at any given SNR. A typical plot of NU 256-QAM constellation is shown in Figure 3 (a).

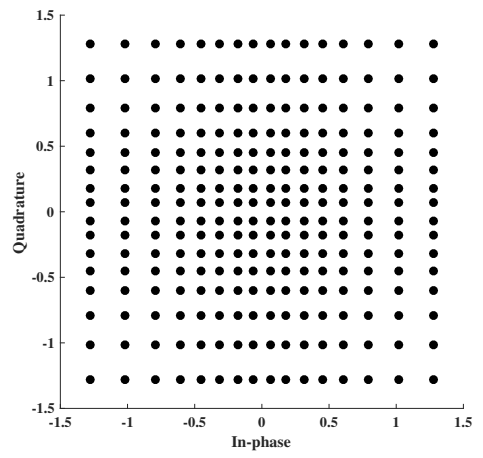

(a) NU 256-QAM

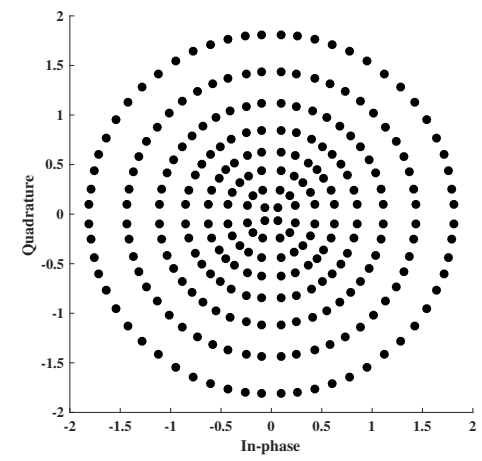

(b) NU 256-QCI

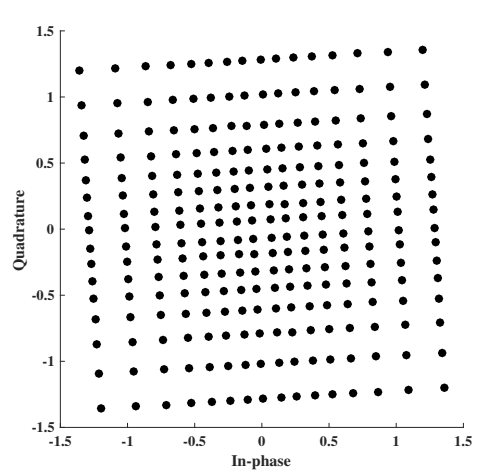

(c) Rotated NU 256-QAM

Figure 3. Optimized 256-QAM constellation and its transforms.

\section{B. Channel-adapted MODCOD transformation}

Having chosen the set of base MODCODs, the next step is to define the functions in the CAT block in order to obtain the MODCODs for other channel models. For non-linear satellite channels, a class of circular constellations has been introduced $i^{7}$ with the achievable mutual information being very close to the peakpower limited channel capacity. This type of constellations, named QCI, is in fact the transformation of QAM points under the radial isomorphism, which converts the concentric squares into concentric rings. Let $(u, v) \in \mathbb{R}^{2}$ denote a QAM constellation point, then the radial mapping is defined as

$$
f_{1}(u, v)=\frac{\sqrt{2} \max (|u|,|v|)}{\sqrt{u^{2}+v^{2}}}(u, v), \quad \text { for }(u, v) \neq(0,0)
$$

where $f_{1}(0,0)=(0,0)$. It is important to notice that the binary Gray labeling of QAM constellation is preserved under $f_{1}$, and therefore the resulting QCI constellation has also a Gray labeling. Using this isomorphism, for each $M$-QAM constellation, a unique $M$-QCI constellation can be constructed.

The radial mapping in Eq. (1) can also be applied to NU QAM signal set. The result will be a QCI constellation with concentric rings of non-uniform radii (see Figure 3 (b)). Even though the resulting QCI constellations are not optimal over the non-linear channel, as we will see in Section III, they perform very close to the state of the art. We refer to these constellations as NU QCI in the rest of this paper.

In addition to non-linear channels, we are also interested in multipath fading channels. An effective solution to increase the robustness of the receiver in severe fading scenarios is shown to apply a certain rotation to the constellation points. ${ }^{9}$ This technique introduces signal space diversity that can achieve 
substantial coding gain over fading channels without spending additional power or bandwidth; the only drawback is higher detection complexity. ${ }^{9,21}$ The simplest form of rotation is given by the function:

$$
f_{2}(u, v)=e^{j \theta}(u, v),
$$

where the rotation angle $\theta$ needs to be optimized depending on the modulation type and order. The constellation rotation in Eq. (2) is followed by an interleaver through a cyclic delay of quadrature components (Q-delay). Therefore, the in-phase and quadrature components of a transmitted symbol are sent via different carriers and time slots, and thus are affected by independent fadings. As a result, either the quadrature or the in-phase component can be used to recover the information. ${ }^{22}$ The constellation rotation and cyclic Q-delay (RQD) technique has been adopted in the DVB-T2 standard. ${ }^{2}$ The transformation of NU 256-QAM constellation under a rotation angle of atan $(1 / 16)$ degrees is illustrated in Figure 3 (c). It should be noted that the meaning of ACM in DVB-T2 is different from that in DVB-S2X. In DVB-T2, the MODCOD in each physical layer pipe (PLP) will be constant in time, but it may be statically reconfigured. In fact, ACM allows PLPs of the entire DVB-T2 signal to use different MODCODs.

As an example for a possible design, we consider the CAT block containing the transformations in Eq. 1 and Eq. 2, i.e.,

$$
\text { CAT }: \begin{cases}(u, v) \rightarrow(u, v) & \text { for linear channel } \\ (u, v) \rightarrow f_{1}(u, v) & \text { for non-linear channel } \\ (u, v) \rightarrow f_{2}(u, v) & \text { for fading channel }\end{cases}
$$

Such a CAT block enables the base MODCOD set to be adapted to use over non-linear and fading channels by a simple transformation of symbols. This will not only reduce the number of MODCODs needed but also allow for lower transmitter and receiver complexities and decrease the amount of MODCOD signaling overhead.

The definition in Eq. (3) can be regarded as one possible realization of the unified ACM scheme; in general, one may define extra criteria to extend the compatibility of CAT with a diverse range of wireless applications.

\section{Mutual Information Analysis}

An appropriate measure for constellation performance is its input-output mutual information. In particular, we are interested in pragmatic communication systems, where symbol demapping is completely decoupled from channel decoding with an interleaver placed in between. The resulting system is called bit-interleaved coded modulation $(\mathrm{BICM})^{23}$ and the relevant measure is BICM capacity, also known as PAMI, which is defined $\mathrm{as}^{24}$

$$
\operatorname{PAMI}(\chi, \mu)=\sum_{i=1}^{M} I\left(\mu^{i}(x) ; y\right),
$$

where $\chi$ and $x$ are respectively the constellation set and its constituting points, $\mu$ is the symbol labeling with $\mu^{i}(x)$ representing the $i$-th bit of the label assigned to $x$, and $y$ denotes the received signal at the output of the channel.

Assuming a memoryless ideal non-linearity model (hard limiter) for the HPA, and ignoring the effect of filters, lead us to set the unit maximum power constraint for the constellations ${ }^{25}$ when employed over such channels. As a result, we calculate the PAMI as a function of PSNR.

In the following, we compare the mutual information of a number of possible MODCODs in the proposed unified ACM with some of the MODCODs used in DVB-S2X and DVB-T2 standards, where PAMI is plotted versus SNR (linear channel) and PSNR (non-linear channel). The PAMIs over the non-linear channel are compared with the capacity of peak-power constrained channels derived by Shamai et al. in. ${ }^{26}$ We consider the base MODCODs with NU QAM constellations of $M=16, M=64$ and $M=256$ points optimized 
for target SNRs of $10 \mathrm{~dB}, 15 \mathrm{~dB}$ and $20 \mathrm{~dB}$, respectively. For each constellation size, only the DVB-S2X MODCOD designed for the same range of SNR (or PSNR) with highest PAMI over the linear (or nonlinear) channel is considered. We adopt the notation introduced $\mathrm{in}^{1}$ for the DVB-S2X MODCODs. For instance, 64-APSK 132/180 refers to the MODCOD designed for the non-linear channel with LDPC code identifier 132/180, and 64-APSK 128/180-L is the MODCOD designed for the linear channel with LDPC code identifier 128/180.

\section{A. 16-ary Constellations}

Figure 4 compares the PAMI of various constellations with 16 points. For the linear channel in Figure 4 (a), there is a slight difference (always below 0.05 bits/symbol in the depicted range of SNR) between the PAMI of 16-APSK 20/30-L constellation and that of NU 16-QAM. As we will see in Section IV, this results in NU 16-QAM being very close to 16-APSK 20/30-L MODCOD in performance. From the same figure, it can also be seen that NU 16-QAM has higher PAMI than 16-QAM which is clearly the result of optimization over the linear AWGN channel. However, this difference is not significant because there is only one tunable parameter in the optimization, i.e., the ratio between the two corresponding positive PAM amplitudes. As for the non-linear channel, the achievable mutual information of 16-APSK 13/18 is slightly higher than the NU 16-QCI constellation, as depicted in Figure 4 (b). Notice that NU 16-QCI constellation performs very close to 16-QCI.

In order to have a comprehensive understanding of the plots in Figure 4, we summarize the results in Table 1. In this table, we provide the comparison of the sum capacity gap and the overall loss with respect to the capacity limits for four different MODCOD pairs. For each pair of MODCODs (the first two columns), the sum capacity gap is obtained by adding the PAMI loss of the linear MODCOD with respect to the Shannon limit and the PAMI loss of the non-linear MODCOD with respect to the Shamai limit for a given SNR value. Similarly, the Overall loss is computed by adding the SNR loss of linear and non-linear MODCODs with respect to the corresponding capacity limits for a fixed value of PAMI.

As it can be seen in Table 1, the best result is obtained by selecting two different MODCODs (DVBS2X MODCODs), each targeting the corresponding channel. However, this gain for DVB-S2X MODCODs is achieved by using two MODCODs, as opposed to only one MODCOD in the CAT scheme. The sum capacity gap and overall loss of NU 16-QAM and NU 16-QCI pair are smaller than those obtained by using a single MODCOD over both the channels.

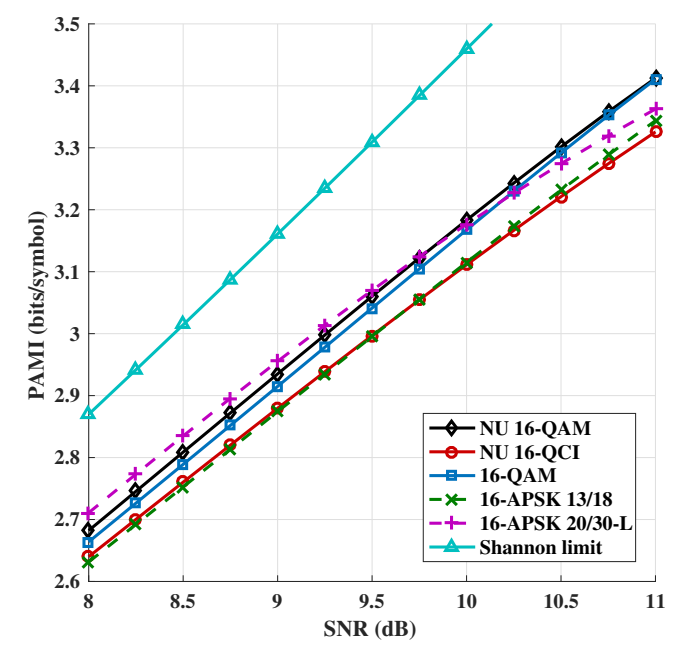

(a) Linear channel

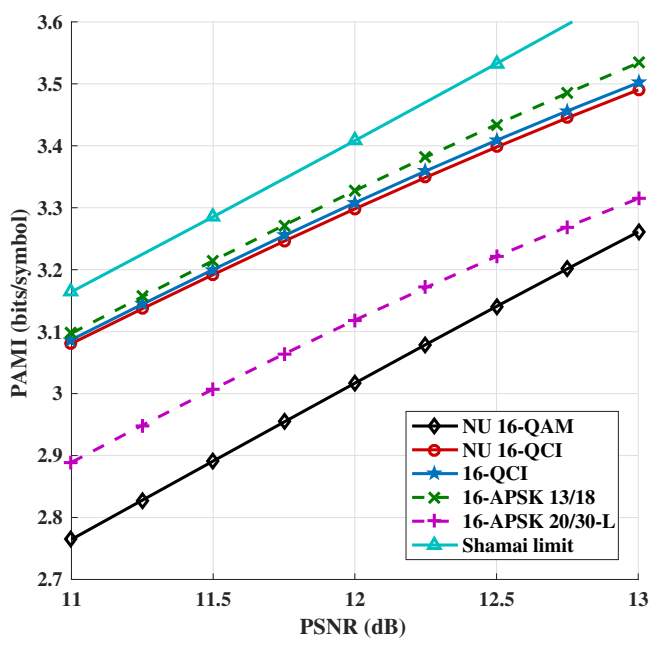

(b) Non-linear channel

Figure 4. PAMI comparison for 16-ary constellations. 
Table 1. Numerical comparison of sum capacity gap and overall loss for the MODCODs with 16-ary constellations. The capacity gaps are obtained for fixed $\mathrm{SNR}=\mathbf{8 . 4 3} \mathrm{dB}$ (linear channel) and PSNR $=11.52 \mathrm{~dB}$ (non-linear channel), and the losses with respect to the capacity limits are calculated for fixed PAMI=2.818 bits/symbol (linear channel) and PAMI=3.219 bits/symbol (non-linear channel).

\begin{tabular}{ccccc}
\hline \multicolumn{2}{c}{ MODCODs } & $\begin{array}{c}\text { Sum capacity gap } \\
(\text { bits/symbol })\end{array}$ & $\begin{array}{c}\text { Overall loss } \\
(\mathrm{dB})\end{array}$ & Notes \\
\cline { 1 - 2 } Linear & Non-linear & & & \\
\hline 16-APSK 20/30-L & 16-APSK 20/30-L & 0.45 & 1.85 & 1 MODCOD \\
16-APSK 13/18 & 16-APSK 13/18 & 0.39 & 1.22 & 1 MODCOD \\
16-APSK 20/30-L & 16-APSK 13/18 & 0.24 & 0.88 & 2 MODCODs \\
NU 16-QAM & NU 16-QCI & 0.29 & 1.08 & CAT scheme \\
\hline
\end{tabular}

\section{B. 64-ary Constellations}

For 64-ary constellations, the comparison of PAMI is drawn in Figure 5. It can be seen from Figure 5 (a) that the PAMI curve of 64-APSK 128/180-L is just over that of NU 64-QAM, with a maximum distance of less than 0.05 bits/symbol in the desired range of SNR. Furthermore, similar to NU 16-QAM, NU 64-QAM also outperforms 64-QAM but with higher PAMI differences. Over the non-linear channel (Figure 5 (b)), using NU 64-QCI achieves almost the same PAMI compared to the best DVB-S2X constellation (64-APSK 132/180 MODCOD).

The results are summarized in Table 2. The difference between the capacity gap of CAT-related MODCODs and the two DVB-S2X MODCODs (each to be used over the targeted channel) is about 0.03 bits/symbol.

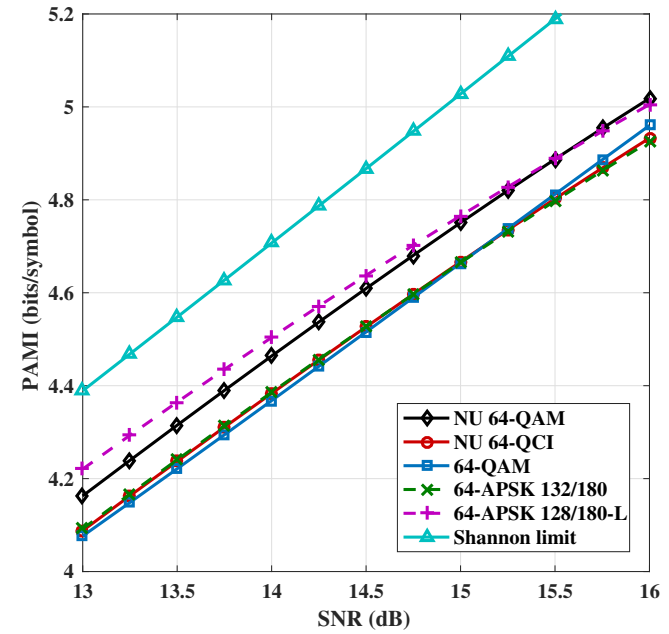

(a) Linear channel

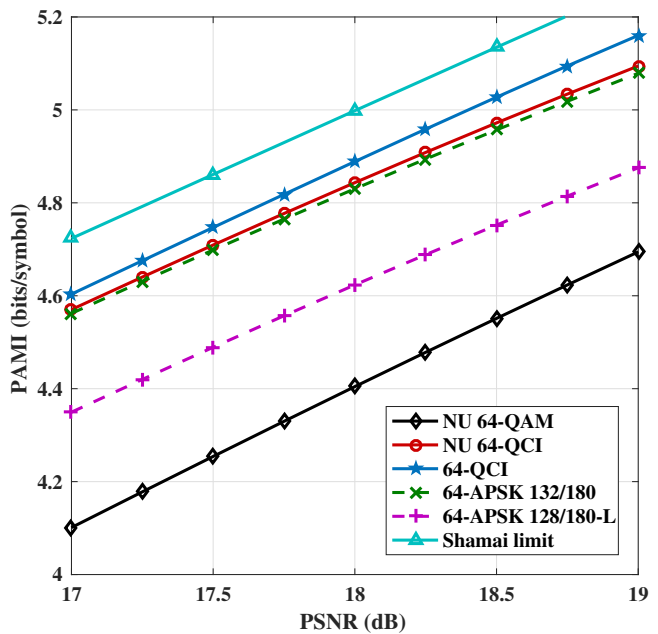

(b) Non-linear channel

Figure 5. PAMI comparison for 64-ary constellations.

\section{256-ary Constellations}

The PAMI comparison for various 256-ary constellations is shown in Figure 6. As it can be observed in Figure 6 (a), over the linear channel, the constellation of 256-APSK 22/30-L MODCOD achieves about 0.1 bits/symbol higher PAMI than NU 256-QAM. Over the non-linear channel, 256-APSK 135/180 and NU 256QCI both achieve the same PAMI in the desired SNR range, as shown in Figure 6 (b). As a consequence, in comparison with the DVB-S2X MODCODs, employing the CAT-related MODCODs of the unified ACM does not lead to significant performance loss in terms of achievable spectral efficiency. The numerical comparison of the four MODCOD pairs is shown in Table 3. The overall conclusion is similar to the previous cases; the 
Table 2. Numerical comparison of sum capacity gap and overall loss for the MODCODs with 64-ary constellations. The capacity gaps are obtained for fixed SNR=13.98 dB (linear channel) and PSNR=17.97 $\mathrm{dB}$ (non-linear channel), and the losses with respect to the capacity limits are calculated for fixed PAMI=4.498 bits/symbol (linear channel) and PAMI=4.822 bits/symbol (non-linear channel).

\begin{tabular}{ccccc}
\hline \multicolumn{2}{c}{ MODCODs } & $\begin{array}{c}\text { Sum capacity gap } \\
(\text { bits/symbol })\end{array}$ & $\begin{array}{c}\text { Overall loss } \\
(\mathrm{dB})\end{array}$ & Notes \\
\cline { 1 - 3 } Linear & Non-linear & & & \\
\hline 64-APSK 128/180-L & 64-APSK 128/180-L & 0.58 & 2.06 & 1 MODCOD \\
64-APSK 132/180 & 64-APSK 132/180 & 0.53 & 1.67 & 1 MODCOD \\
64-APSK 128/180-L & 64-APSK 132/180 & 0.38 & 1.25 & 2 MODCODs \\
NU 64-QAM & NU 64-QCI & 0.40 & 1.34 & CAT scheme \\
\hline
\end{tabular}

loss due to using the CAT scheme is about $0.27 \mathrm{~dB}$ when compared to the 2-MODCOD DVB-S2X scenario.

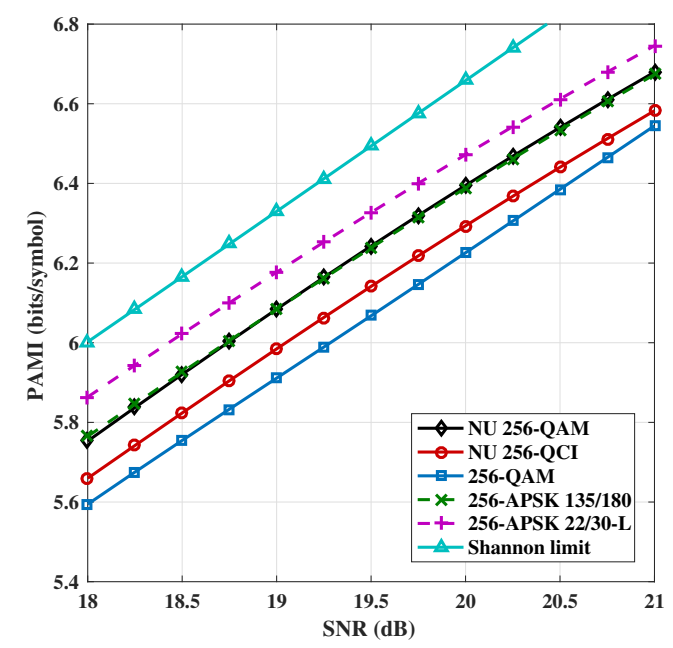

(a) Linear channel

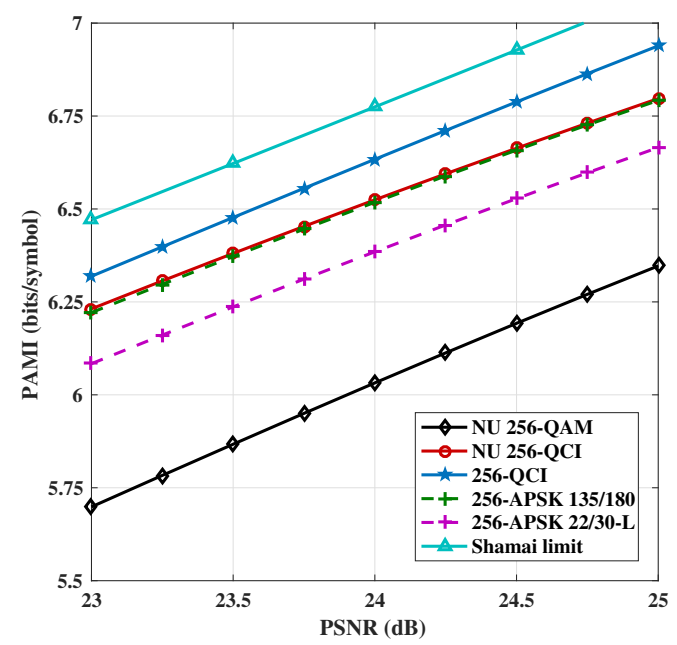

(b) Non-linear channel

Figure 6. PAMI comparison for 256-ary constellations.

\section{Simulation Results}

In this section, we present the BER simulation results for all the constellations analyzed in the previous section. The results are obtained over various channel models according to DVB-S2X and DVB-T2 scenarios. For DVB-S2X, the simulations are performed over both linear and non-linear channels. Comparisons under the DVB-T2 scenario are drawn assuming an i.i.d Rayleigh fading channel.

In all simulations, we assume a simple communication chain where the information bits are encoded via an LDPC encoder, modulated and then passed through the channel. We also assume a pragmatic receiver where the demodulator computes log-likelihood ratio (LLR) value for each bit and then passes it on to the LDPC decoder, and no iteration occurs between them. In order to have a fair comparison, we select the same LDPC code of rate 3/4 from DVB-S2X standard. ${ }^{1}$ This coding rate is so selected to be as close to the LDPC code identifiers of all the DVB-S2X MODCODs of interest as possible. For the other MODCODs than DVB-S2X, we drop the code identifier 3/4; for instance, NU 16-QAM MODCOD (or NU 16-QAM for short) refers to the combination of NU 16-QAM and the selected LDPC code with rate $3 / 4$.

\section{A. Comparison over DVB-S2X channel models}

Similar to the previous section, the simulation results are categorized based on the modulation order. As we will see, the BER results confirm the theoretical studies of PAMI in all cases. 
Table 3. Numerical comparison of sum capacity gap and overall loss for the MODCODs with 256-ary constellations. The capacity gaps are obtained for fixed SNR=18.84 dB (linear channel) and PSNR=24.02 $\mathrm{dB}$ (non-linear channel), and the losses with respect to the capacity limits are calculated for fixed PAMI=6.128 bits/symbol (linear channel) and PAMI=6.524 bits/symbol (non-linear channel).

\begin{tabular}{ccccc}
\hline \multicolumn{2}{c}{ MODCODs } & $\begin{array}{c}\text { Sum capacity gap } \\
\text { (bits/symbol })\end{array}$ & $\begin{array}{c}\text { Overall loss } \\
(\mathrm{dB})\end{array}$ & Notes \\
\cline { 1 - 2 } Linear & Non-linear & & & \\
\hline 256-APSK 22/30-L & 256-APSK 22/30-L & 0.54 & 1.77 & 1 MODCOD \\
256-APSK 135/180 & 256-APSK 135/180 & 0.51 & 1.61 & 1 MODCOD \\
256-APSK 22/30-L & 256-APSK 135/180 & 0.40 & 1.31 & 2 MODCODs \\
NU 256-QAM & NU 256-QCI & 0.49 & 1.58 & CAT scheme \\
\hline
\end{tabular}

\section{MODCODs with 16-ary Constellations}

Figure 7 compares the BER performance of various MODCODs that use 16-ary constellations. As it can be seen in Figure 7 (a), the performance of NU 16-QAM over the linear channel is quite close to 16-APSK 20/30-L and superior to 16-QAM. Over the non-linear channel (Figure 7 (b)), NU 16-QCI shows a slight loss (less than $0.1 \mathrm{~dB}$ ) with respect to 16-APSK 13/18 MODCOD. We can further see that the difference between NU 16-QCI and 16-QCI is insignificant.

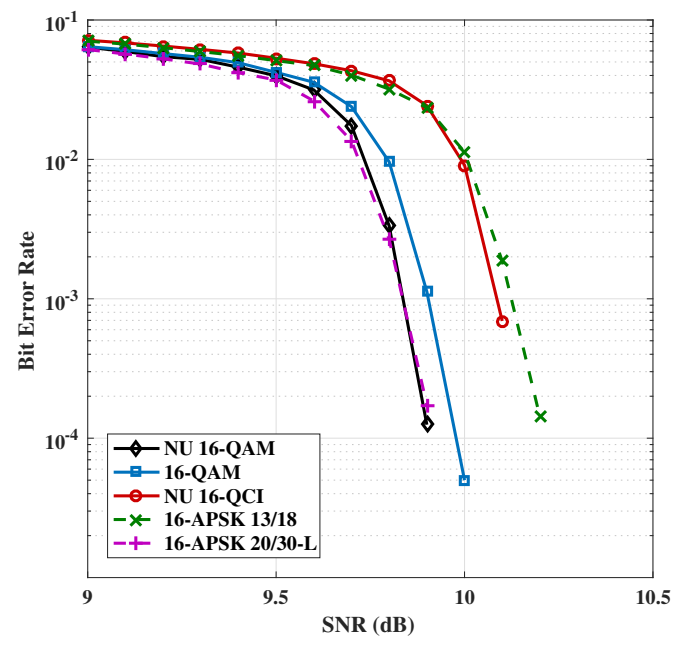

(a) Linear channel

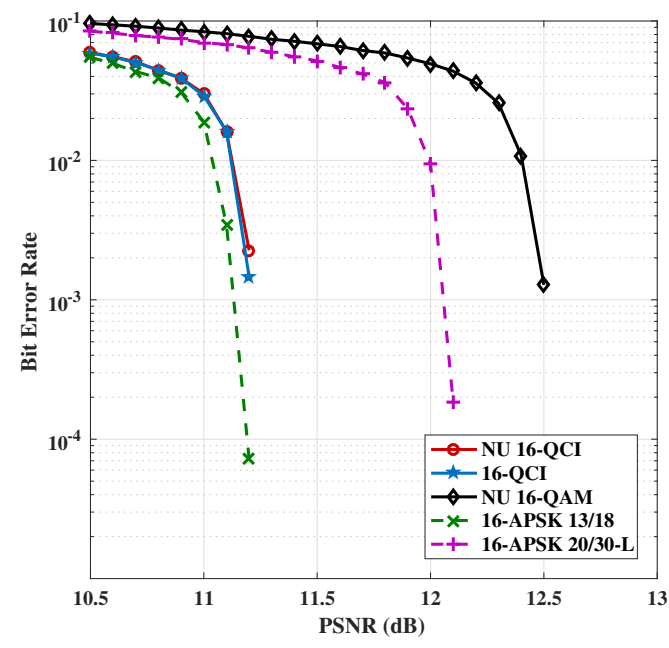

(b) Non-linear channel

Figure 7. BER performance of the MODCODs with 16-ary constellations.

\section{MODCODs with 64-ary Constellations}

For MODCODs with 64-ary constellations, the BER curves are plotted in Figures 8 (a) and 8 (b) for linear and non-linear channels, respectively. As before, the BER simulations confirm closely the PAMI results. A loss of $0.1 \mathrm{~dB}$ is resulted from using NU 64-QAM compared to 64-APSK 128/180-L MODCOD. But over the non-linear channel, using the NU 64-QCI MODCOD provides a small gain compared to 64-APSK 132/180.

\section{MODCODs with 256-ary Constellations}

The BER performance of various MODCODs with 256-ary constellations is depicted in Figure 9. As it can be seen from Figure 9 (a), NU 256-QAM MODCOD shows a performance loss of about $0.25 \mathrm{~dB}$ compared to 256-APSK 22/30-L, but a gain of $0.5 \mathrm{~dB}$ against the uniform 256-QAM. As for the non-linear channel, Figure 9 (b) shows that NU 256-QCI performs around $0.1 \mathrm{~dB}$ better than the best DVB-S2X non-linear MODCOD in this SNR range (256-APSK 138/180). It follows from the BER results that the CAT-related 


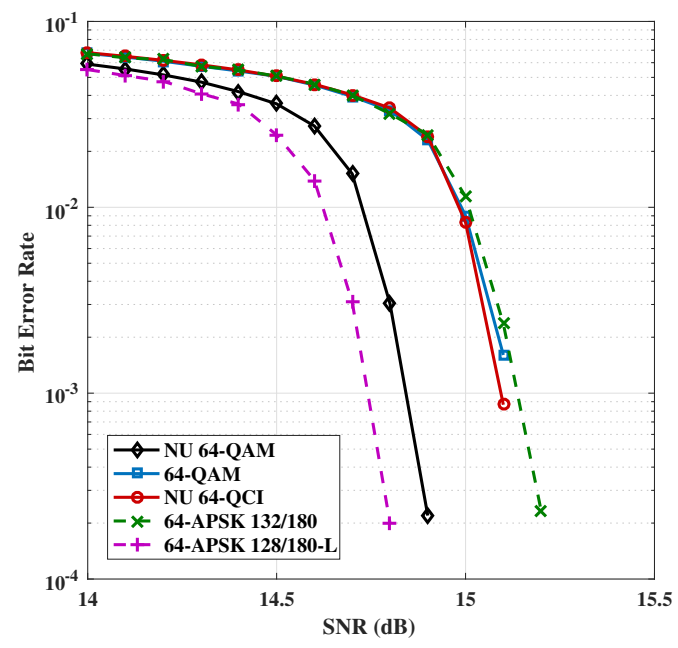

(a) Linear channel

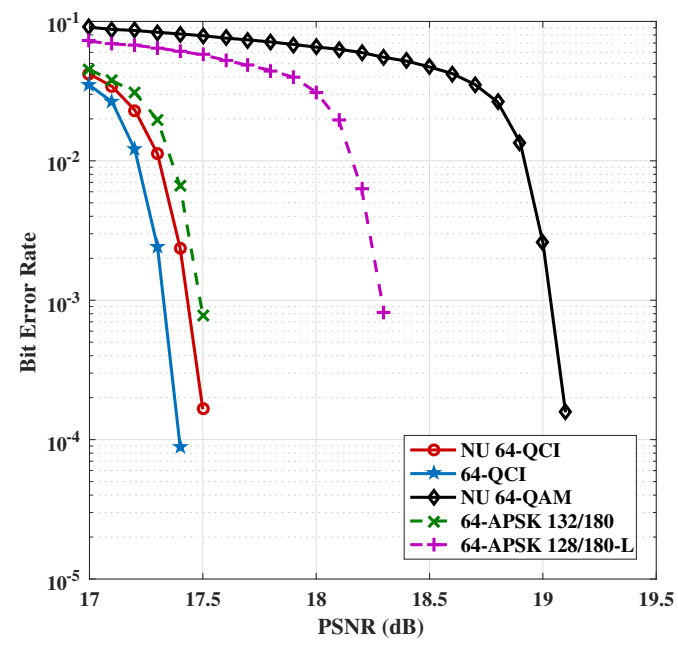

(b) Non-linear channel

Figure 8. BER performance of the MODCODs with 64-ary constellations.

MODCODs provide competitive performance with respect to the separate linear and non-linear MODCODs of DVB-S2X, which is in line with the PAMI results obtained in the previous section.

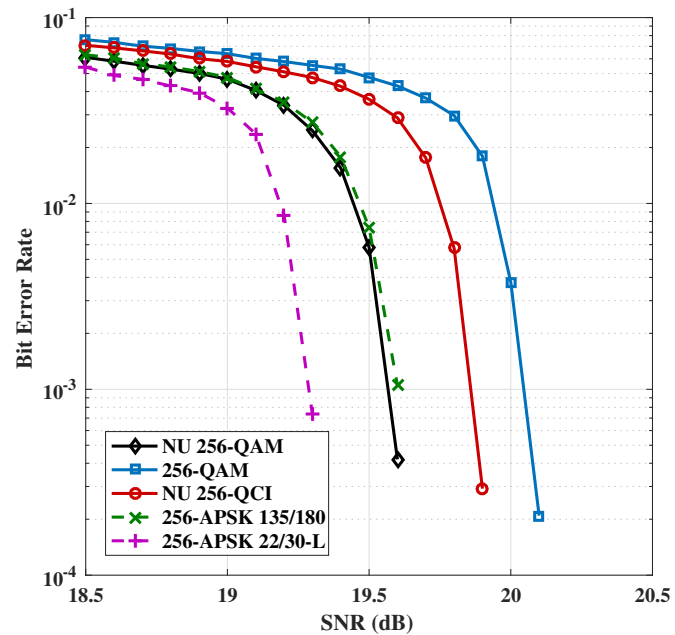

(a) Linear channel

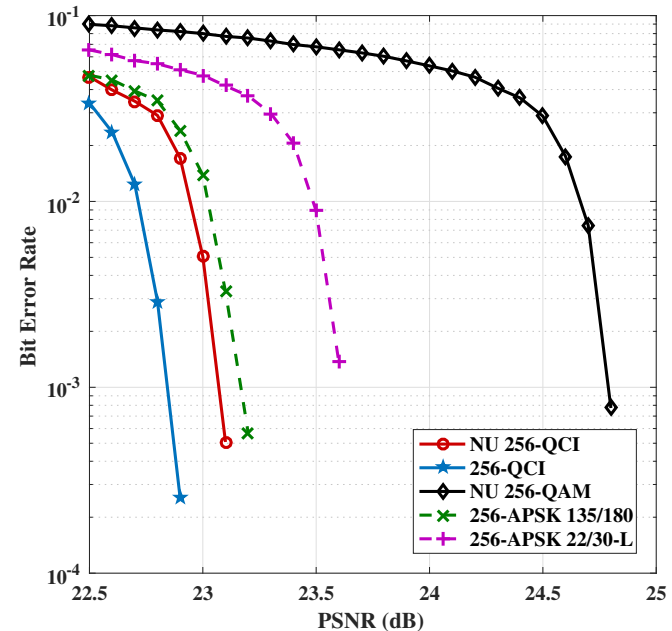

(b) Non-linear channel

Figure 9. BER performance of the MODCODs with 256-ary constellations.

\section{B. Comparison over DVB-T2 channel model}

In our simulations over the i.i.d. Rayleigh channel we consider the data transmission both with and without RQD. The BER results are depicted in Figure 10. We adopt the same rotation angle as in DVB-T2 standard for each modulation order. As mentioned earlier in Section II, the RQD technique provides higher probability of successful detection for deeply faded symbols. Without RQD, the BER performance is substantially degraded, falling into the error floor region at rather high BER. Comparing with the BER results over the AWGN channel, using RQD reduces the fade margin from $8 \mathrm{~dB}$ (for the non-rotated constellations) to $3 \mathrm{~dB}$ (at $10^{-2}$ ) over the assumed Rayleigh channel.

Furthermore, the BER results show that NU QAM performs better than QAM over fading channels for 
all the constellation sizes of interest. We also simulate the BER of the best linear DVB-S2X MODCOD for each constellation size. All the considered DVB-S2X linear MODCODs show a loss with respect to both QAM and NU QAM, even if RQD is employed. As one other possible scenario for APSK constellations, we applied the cyclic Q-delay without constellation rotation. The BER results in that case were slightly worse than those obtained with RQD, and thus are not presented here.

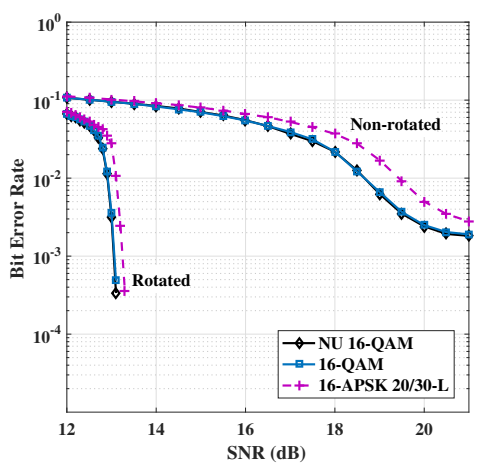

(a) 16-ary

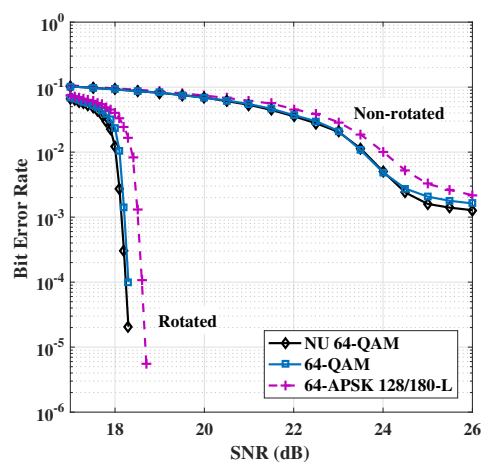

(b) 64-ary

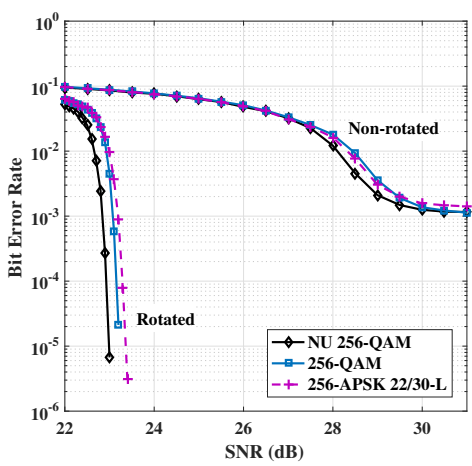

(c) 256-ary

Figure 10. BER performance over the Rayleigh fading channel with and without RQD.

A numerical performance comparison is presented in Table 4, where the approximate gains of the NU QAM constellations with respect to DVB-T2 and DVB-S2X constellations over the Rayleigh channel are shown. Both the rotated and non-rotated constellations with 16, 64 and 256 points are considered. NU QAM has always better or equal BER performance with respect to the QAM constellation. Moreover, NU QAM shows performance gains up to $1 \mathrm{~dB}$ compared to the DVB-S2X MODCOD in all cases. It should be noted that the gains in Table 4 are obtained using the optimal rotation angles for uniform QAM constellations. However, if one optimizes the rotation angle for each NU QAM constellation, even higher gains might be achievable. ${ }^{13}$

Table 4. Performance gain of the proposed ACM scheme with respect to the two broadcasting standards over the Rayleigh fading channel. All the gains are computed at $\mathrm{BER}=10^{-2}$.

\begin{tabular}{ccc|cc}
\hline & \multicolumn{2}{c}{ Rotated } & \multicolumn{2}{c}{ Non-rotated } \\
\cline { 2 - 5 } Modulation order & DVB-T2 & DVB-S2X & DVB-T2 & DVB-S2X \\
\hline 16 & $0 \mathrm{~dB}$ & $0.2 \mathrm{~dB}$ & $0 \mathrm{~dB}$ & $1 \mathrm{~dB}$ \\
64 & $0.1 \mathrm{~dB}$ & $0.4 \mathrm{~dB}$ & $0 \mathrm{~dB}$ & $0.5 \mathrm{~dB}$ \\
256 & $0.25 \mathrm{~dB}$ & $0.4 \mathrm{~dB}$ & $0.5 \mathrm{~dB}$ & $0.4 \mathrm{~dB}$ \\
\hline
\end{tabular}

\section{Conclusions and Further Research}

In this paper we propose a new ACM scheme which can potentially unify the MODCOD design for several existing standards. This is done by introducing a new block, called channel-adapted transformation (CAT), to the communication chain. The functionality of CAT is to adapt an existing MODCOD to the given new channel condition through a deterministic transformation. As a result, the MODCODs designed for the linear channel can also be used over non-linear or fading channels by passing them through the CAT block. Another consequence of introducing the CAT block is to reduce the number of existing MODCODs and hence reducing the length of physical layer header. Our simulation results show that the overall loss with respect to DVB-S2X, where MODCODs are separately designed for both linear and nonlinear channels, is around $0.2 \mathrm{~dB}$ in the worst case. 


\section{Acknowledgments}

The authors would like to thank Bhavani Shankar and Björn Ottersten for several discussions and comments.

The authors are supported by the Luxembourg National Research Fund under CORE Junior project: C16/IS/11332341 Enhanced Signal Space opTImization for satellite ComMunication Systems (ESSTIMS).

\section{References}

${ }^{1}$ Digital Video Broadcasting (DVB); Second generation framing structure, channel coding and modulation systems for Broadcasting, Interactive Services, News Gathering and other broadband satellite applications; Part 2: DVB-S2 Extensions (DVB-S2X), ETSI EN Std. 302 307-2 V1.1.1, Oct. 2014.

${ }^{2}$ Digital Video Broadcasting (DVB); Frame structure channel coding and modulation for a second generation digital terrestrial television broadcasting system (DVB-T2), ETSI EN Std. 302755 V1.3.1, Apr. 2012.

${ }^{3}$ CCSDS protocols over DVB-S; Summary of definition, implementation and performance, CCSDS 130.12-G-1, Nov. 2016.

${ }^{4}$ R. De Gaudenzi, A. Guillen i Fabrigas and A. Martinez, "Performance analysis of turbo-coded APSK modulations over nonlinear satellite channels," IEEE Trans. Wirel. Commun., vol. 5, pp. 23962407, 2006.

${ }^{5}$ H. Meric, "Approaching the Gaussian Channel Capacity With APSK Constellations," IEEE Commun. Lett., vol. 19, no. 7, pp. 1125-1128, July 2015. 2016 .

${ }^{6}$ N. Loghin et al., "Non-uniform constellations for ATSC 3.0," IEEE Trans. Broadcasting, vol. 62, no. 1, pp. 197-203, Mar.

${ }^{7}$ F. Kayhan, "QAM to circular isomorphic constellations," in Proc. Advanced Satellite Multimedia Syst. Conf., Palma de Mallorca, Spain, 2016.

${ }^{8}$ F. Kayhan, "High Order APSK Constellation Design for Next Generation Satellite Communication," in Proc. AIAA Int. Commun. Satellite Syst. Conf. (ICSSC), Cleveland, USA, 2016.

${ }^{9} \mathrm{~J}$. Boutros and E. Viterbo, "DVB-T2: Signal space diversity: A power- and bandwidth-efficient diversity technique for the Rayleigh fading channel," IEEE Trans. Inform. Theory, vol. 44, no. 4, pp. 1453-1467, Jul. 1998.

${ }^{10}$ R. De Gaudenzi and Rita Rinaldo, "Adaptive coding and modulation for next generation broadband multimedia systems," in Proc. AIAA Satellite Commun. Syst. Conf., Montreal, May 2002.

${ }^{11}$ G. Gardikis, N. Zotos, and A. Kourtis, "Satellite media broadcasting with adaptive coding and modulation," Int. J. Digital Multimedia Broadcasting, Sep. 2009.

${ }^{12}$ R. De Gaudenzi, A. G. i Fabregas, A. Martinez, and B. Ponticelli, "High power and spectral efficiency coded digital modulation schemes for nonlinear satellite channels," in Proc. Int. Workshop Digital Signal Process. Techn. for Space Commun., Sesimbra, Portugal, 2011.

${ }^{13}$ J. Zoellner and N. Loghin, "Optimization of high-order non-uniform QAM constellations," in Proc. IEEE Int. Symp. Broadband Multimedia Syst. and Broadcasting, London, 2013.

${ }^{14}$ D. Sommer and G. P. Fettweis, "Signal shaping by non-uniform QAM for AWGN channels and applications using turbo coding," ITG FACHBERICH, pp. 81-86, Jan. 2000.

${ }^{15}$ B. Mouhouche, D. Ansorregui, and A. Mourad, "High order non-uniform constellations for broadcasting UHDTV," in Proc. IEEE Wirel. Commun. and Netw. Conf., Istanbul, Turkey, 2014.

${ }^{16}$ J. Hossain, A. Alvarado, and L. Szczecinski, "BICM Transmission Using Non-Uniform QAM Constellations: Performance Analysis and Design," in Proc. IEEE Int. conf. Commun., Cape Town, South Africa, 2010.

${ }^{17}$ ATSC Standard, Physical Layer Protocol, Doc. A/322:2017, Jun. 2017.

${ }^{18}$ G. J. Foschini, R. D. Gitlin, and S. B. Weinstein, "Optimization of two-dimensional signal- constellations in the presence of Gaussian noise," IEEE Trans. Commun., vol. 22, no. 1, pp. 28-38, Jan. 1974.

${ }^{19}$ G. Forney, R. Gallager, G. Lang, F. Longstaff, and S. Qureshi, "Efficient modulation for band-limited channels," IEEE J. Sel. Areas Commun., vol. 2, no. 5, pp. 632-647, Sep. 1984.

${ }^{20}$ F. Kayhan and G. Montorsi, "Constellation design for memoryless phase noise channels," IEEE Trans. Wirel. Commun., vol. 13, no. 5, pp. 2874-2883, May 2014.

${ }^{21} \mathrm{M}$. Li, C. Abdel-Nour, C. Jego, and C. Douillard, "Design of rotated QAM mapper/demapper for the DVB-T2 standard" in Proc. IEEE Workshop Signal Process. Syst., Tampere, Finland, 2009.

${ }^{22}$ D. Perez-Calderon, V. Baena-Lecuyer, A. C. Oria, P. Lopez, and J. G. Doblado, "Rotated constellation demapper for DVB-T2," Electron. Lett., vol. 47, no. 1, pp. 31-32, Jan. 2011.

${ }^{23}$ G. Caire, G. Taricco, and E. Biglieri, "Bit-interleaved coded modulation," IEEE Trans. Inform. Theory, vol. 44, no. 3, pp. 927-946, May 1998.

${ }^{24}$ G. Caire, G. Taricco, and E. Biglieri, "Capacity of bit-interleaved channels," Electron. Lett., vol. 32, no. 12, pp. 1060-1061, Jun. 1996.

${ }^{25}$ F. Kayhan and G. Montorsi, "Constellation design for transmission over non-linear satellite channels," in Proc. IEEE Glob. Telecommun. Conf., Annaheim, USA, 2012.

${ }^{26} \mathrm{~S}$. Shamai and I. Bar-David, "The capacity of average and peak-power-limited quadrature Gaussian channels," IEEE Trans. Inform. Theory, vol. 41, no. 4, pp. 1060-1071, Jul. 1995. 